\title{
Neural Networks in ERP and CRM
}

\author{
Mary. A.S. \\ Research scholar \\ karunya University \\ Coimbatore
}

\author{
P. Ranjit Jeba Thangaiah \\ Assistant Professor [SG] \\ Department of Computer Applications \\ Karunya University, Coimbatore
}

\begin{abstract}
Enterprise Resource Planning (ERP) is a very popular term nowadays which integrates all the major functions like Finance, Controlling, Production, Selling and Distribution, Personnel, Quality Control, Material management of the concern. ERP is applicable both in big and medium size industries. CRM mainly concentrates to satisfy the consumers at a maximum level. Data mining is a computer technique which helps to coordinate these two parts through the way of applying best algorithm and deriving the results. This research paper uses neural networks for obtaining customer value as well as product value for a specific customer or product. Then these customer or product values are to be combined into clusters by using K-Mean algorithm. The testing results prove that this method gives more accuracy than Naïve Bayes and Decision tree J-48 classification techniques. Experimental results show a satisfactory performance. The results obtained from this research work helps the organization to find out a most suitable marketing strategy in the near future.
\end{abstract}

\section{KEYWORDS}

Enterprise Resource Planning, Customer relationship management, Naïve Bayes, Neural networks

\section{INTRODUCTION}

Nowadays most of the companies use ERP system because huge amount of data can be maintained easily under a single system. It includes all the functions and activities of the companies and maintaining all the related data by a computerized system. ERP is a single system which integrates each and every function of the organization. It helps the concern's growth [4]. ERP software can be used in any type of industry whether it is a manufacturing concern or other type of industry. ERP consists of three parts namely, client server, database and applications. It unites entire function of the company [3]. ERP system outright the older method of keeping data, due to its capacity of handling large volume of data. So most of the giant companies follow ERP system [6] the information related to different functions of the companies is to be stored in this system and they are distributed to different functional models as and when they are needed. Some ERP systems have web-based interfaces. ERP is used in each area of companies' functional activity and enhances the efficiency of each function like material management, customer relationship management, production, quality control and inspection, marketing etc.[8]. ERP solves all the departmental problems of the companies [5].

ERP includes all the business functions like Planning, Purchasing, Capacity planning, Production, Quality control, Inspection, Maintenance, selling and distribution, Finance, procurement, Marketing etc. The entire organization data is stored in only one database which is known as manufacturing or central database [15]. CRM is one of the business functions which help to gain new customers as well as retaining the existing customers. CRM is process oriented, technical, capability based, philosophical and strategic one. CRM investments with the combination of ERP system helps to measure the customer profitability in most of the business organizations [12]. Integrating ERP with CRM increases customer profitability and company's growth[16]. The ERP system benefits can be maximized by uniting all the functions of the organization [15].

\section{CRM}

Customer Relationship Management(CRM)emerges in the early of 1990s. CRM consists of methods and techniques for capturing and maintaining the customers for their organizations [1]. CRM is nothing but creating the customers for the company's products, studying about their needs and wants, finding the ways to satisfy their customers and make customers to aware of their products[7]. CRM helps the companies to maximize their profits and strengthen their positions in the markets against the competitors through maintaining close relationships with their consumers[2]. CRM helps the companies to identify the buying patterns and behavior of their consumers. From this analysis the companies estimate their production and other procurement requirements. CRM helps to gain and retain the customers through business tactics and helps the organizations to maximize the customer value. Companies mostly concentrate to keep up their old customers. It helps the organizations to reduce their cost for attracting new customers[10].

\section{NEURAL NETWORKS}

Neural networks contain lot of information. Each node contains some information. All the nodes are processing the information and producing the final results[13]. Neural networks are applicable to find out the information from a complex pattern[14]. Neuron is a processing unit. It is a node, which processes all the information fed as an input and produces the output according to the activation function. This function is called as an activation function. It may be a linear or nonlinear function, which maps from the input to the output. It is denoted by $\theta$. A neuron is connected with other neuron by variable weights. Research on Neural networks is first done by McCulloch and Pitts in the year of 1940[9]. Neural networks are also called as Connectionists models. Neural networks are very much popular because, it consists of so many neurons and each neuron process the information individually and continuously. Backpropogation algorithm with multilayer perceptron in neural networks is a popular method. It is published in the y ear of 1986 by Rumelhart et al. [11]. 


\section{RESEARCH WORK}

This research work proposes a new method of integrating decisions between Customer Relationship Management and Enterprise Resource Planning activity Production for the purpose of making valuable decisions in Marketing as well as in Production and Sales side by using neural networks a classification technique of data mining.

This research work consists of three phases. First, the necessary data or information like customer name, age, salary, region, religion etc are to be collected from the organization and passing them to the neural networks. Neural networks analyze these properties and combine them with the weight values provided to each perceptron and provide a resultant value. This resulting value is called customer value that corresponds to a specific customer. Every customer data is sent through a similar process to obtain a customer value. The second phase deals with combining these values into clusters using the k-means algorithm. Like this way, the data relating to the products are passed to the neural networks. Neural networks analyze these properties and combine them with the weight values provided to each perceptron and provide a resultant value. This resulting value is called product value that corresponds to a specific product. Every product data is sent through a similar process to obtain a product value. Then these values are combined into clusters using the k-means algorithm. Each cluster (customer) is then analyzed for similarities. All the similar properties are taken as the properties of the entire cluster. For example, all customers in the cluster might have bought mobile phones in the range of 25000 to 50000 . Each product cluster is analyzed and similar properties are determined. In the third phase, new customer data is presented to the system. This data is passed through neural networks to obtain a customer value. This value is then classified into any one of the existing clusters. All the properties that belong to the cluster are assumed to be the properties of the corresponding customer. For example, if the new customer is classified into a cluster with a purchase range of 15000 to 20000 , all the products present in the product cluster that have the range 15000 to 20000 are presented to the customer. Multilayer feed-forward neural networks trained by back-propagation training algorithm is used for classification to find out the desired results. The dataset from Mobile Hand Phone Company is taken to test the prototype for this research.

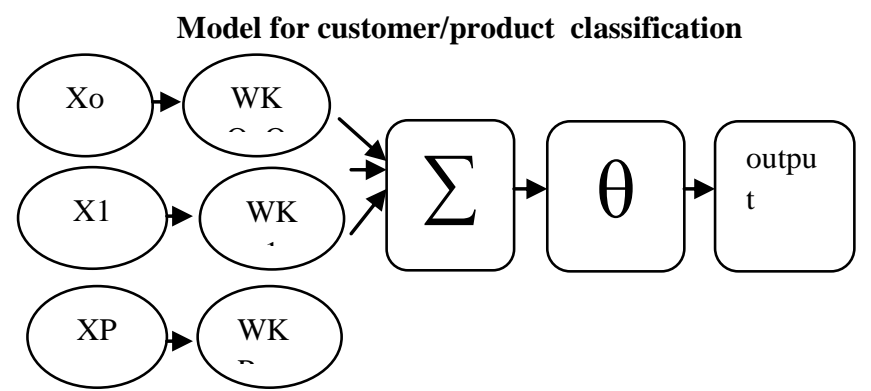

Here X0, X1, XP are the inputs represents Customer's information like their age, salary, income etc as well as product information like brand value, quality, quantity etc.

WKO, WK1, WKP denotes the weights. Weights assignment depends upon the importance of the relative attribute in relation with buying pattern of the customer and sales pattern of the products. $\sum$ represents the potential value of the customer that is the total summation of all inputs and total sales value

$\Theta$ is a threshold value. Various functions of classification are applied on this basis only.

Output provides the final result of various customer classifications and the type of product selection by them for purchasing. In case of Product classification this output provides the details about the product's sales pattern and the range of customers who could purchase the particular type of the product.

\section{EXPERIMENTAL SETUP}

A sample data set of 120 records is taken for testing the results. Each record consists of 13 attributes such as CID, Name ,City, Salary, Area of living, Qualification, Occupation, Type of house, Marital status, Age, No of children and Religion. WEKA tool is used for testing the accuracy.

The following figures shows testing results

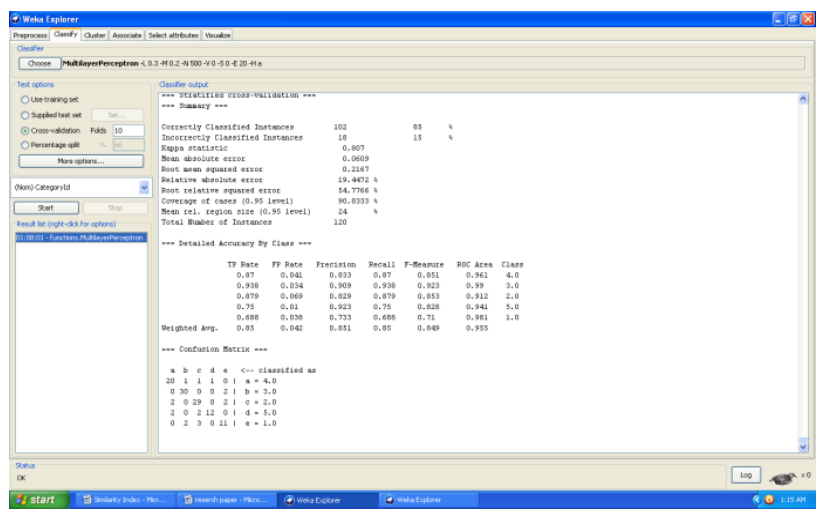

Figure 1: Multilayer Perceptron

The above figure 1 shows that 102 instances with $85 \%$ accuracy are correctly classified and 18 instances are incorrectly classified with $15 \%$. It also shows various statistical errors percentages, detailed accuracy for each c lass and confusion matrix. Ten fold cross validation test mode is used for this testing purpose.

The same data sets are tested on J-48 Decision trees and on Naïve Bayes. The following figures show the results.

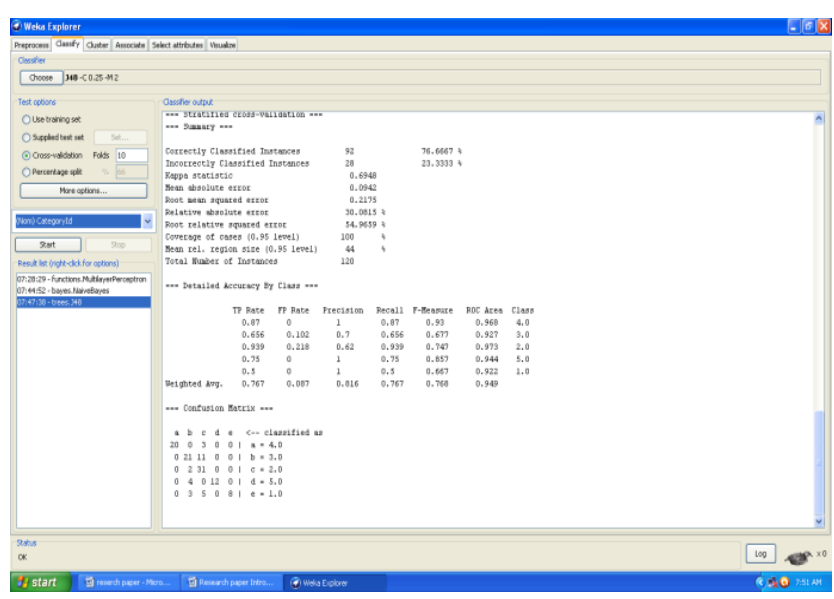

Figure 2: J-48 Decision trees 
The above fig 7 shows that 92 instances are correctly classified with $76.6667 \%$ accuracy and 28 instances are incorrectly classified with $23.3333 \%$.

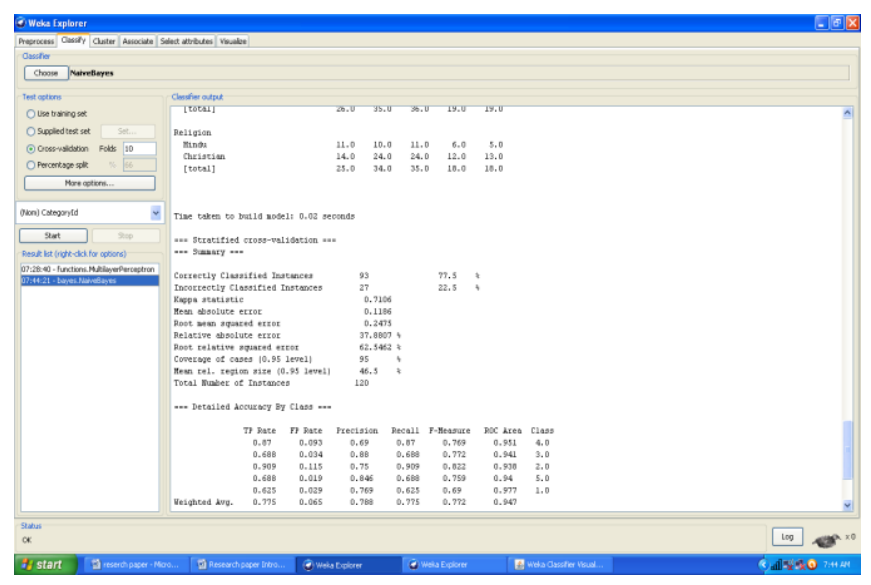

Figure 3: Naïve bayes

Under naïve bayes output summary, 93 instances are correctly classified with $77.5 \%$ accuracy and 27 instances are incorrectly classified with $22.5 \%$.

Table 1: Comparative statement

\begin{tabular}{|c|c|c|}
\hline Multilayer & Naïve bayes & $\begin{array}{ll}\mathrm{J}-48 & \text { Decision } \\
\text { tree } & \\
\end{array}$ \\
\hline $\begin{array}{l}\text { Correctly } \\
\text { classified } \\
\text { instances } 102 \\
85 \%\end{array}$ & $\begin{array}{l}\text { Correctly } \\
\text { classified } \\
\text { instances } 93 \\
77.5 \%\end{array}$ & $\begin{array}{l}\text { Correctly } \\
\text { classified } \\
\text { instances } 92 \\
76.6667 \%\end{array}$ \\
\hline $\begin{array}{l}\text { Incorrectly } \\
\text { classified } \\
\text { instances } 18 \\
15 \%\end{array}$ & $\begin{array}{l}\text { Incorrectly } \\
\text { classified } \\
\text { instances } 27 \\
22.5 \%\end{array}$ & $\begin{array}{l}\text { Incorrectly } \\
\text { classified } \\
\text { instances } 28 \\
23.333 \% \\
23.3333 \%\end{array}$ \\
\hline
\end{tabular}

In both the cases, the accuracy level is lesser than the multilayer perceptron. However, the testing is not done among larger real world data sets. So multilayer feed forward neural networks shows a higher accuracy in testing results.

\section{CONCLUSION}

This Research work helps the organization to find out the most suitable marketing segment and to capture the different needs of the different customers. So it helps the concern to concentrate in those important areas and target its production accordingly in the future. By applying neural networks, we got the most accurate results. Further this research can be extended to other types of manufacturing and selling industries. Other data mining techniques also can be used to reduce the error rate.

\section{REFERENCES}

[1] Bligh, P. and D. Turk, 2004, CRM Unplugged-Releasing CRM's Strategic Value, 1st Edn. John Wiley and Sons, USA., ISBN: 978-0-471-48304-5, pp: 224.

[2] Bueren, A., R. Schierholz, L. Kolbe and W. Brenner, 2004, CKM-improving performance of customer relationship management with knowledge management. Proceeding of the 37th IEEE International Conference on System Sciences, Jan. 5- 8, Hawaii, USA. pp: 1-10.

[3] Davenport, Thomas H. (2000), Mission critical, realizing the Promise of Enterprise Systems, Harvard Business
School Press, Cambridge Mass.

[4] First A. Abdullah S. Al-Mudimigh, Second B. Zahid Ullah, Third C. Farrukh Saleem, A framework of an Automated Data Mining Systems Using ERP Model, International Journal of Computer and Electrical Engineering, Vol. 1, No. 5 December, 2009.

[5]Gail Corbitt, Marinos Themistocleous, Zahir Irani, "ERP/EAI System Issues and Answers: A research Journey", in proceeding: IEEE conference on system sciences, 2005.

[6] Jae-won Park, Nam-Yong Lee, "A Conceptual Model of ERP for Small and Medium-Size Companies Based on UML", IJCSNS International Journal of Computer Science and Network Security, VOL.6 No.5A, May 2006.

[7] Jutla, D., 2001, Enabling and measuring electronic customer relationship management readiness, Proceedings of 34th IEEE Hawaii International Conference on System Sciences, Jan. 3 -6, IEEE Computer Society Washington, DC, USA., pp: 70237032.

[8] McCarthy, Vance. "ERP Gets Down to E-Business." HP World, January 2000.

[9] McCulloch WS, PittsW (1943), A logical calculus of the I deas immanent in nervous activity, Bull of Math Biophysics 5:115-133.

[10] Peppard, J. (2000), Customer relationship management $(\mathrm{CRM})$ in financial services, European Management Journal, 18(3), 312-327.

[11] Rumelhart DE, Hinton GE, Williams RJ (1986), Learning internal representations by error propogation. In:Rumehart DE, McClelland JL (eds) Parallel distributed processing: Explorations in the microstructure of cognition, 1: Foundation, 318-362. MIT Press, Cambridge.

[12] Sonja Grabner-Kraeuter, Martin Waigunyc, Werneer Mussnigb, "Performance Monitoring of CRM Initiatives", in Proceedings: IEEE conference on System Sciences, 2007.

[13] Spangler, W. E., May, J. H., \& Vargas, L. G. (1999), Choosing datamining methods for multiple classification: Representational and performance measurement implications for decision support, Journal of Management Information Systems, 16(1), 37-62.

[14] Venugopal, V., \& Baets, W. (1994), Neural networks and their applications in marketing management. Journal of Systems Management, 45(9), 16-21.

[15] Virgil Chichernea, Romanian, "THE USE OF THE ERPCRM CIM SYSTEMS within THE MASTER'S DEGREE PROGRAMMES" In Proceedings: Annales Universitatis Apulensis Series Oeconomica, Nr.9/2007, Volume 2.

[16] Wen-Hsiung Wu, Chin-Fu Ho, Hsin-Pin Fu, Tien-Hsiang Chang, "SMES IMPLEMENTING AN INDUSTRY SPECIFIC ERP MODEL USING A CASE STUDY APPROACH", Journal of the Chinese Institute of Industrial Engineers, Vol. 23, No. 5, 2006, pp. 423-434. 